\title{
Photon Molecules in Atomic Gases Trapped Near Photonic Crystal Waveguides
}

\author{
James S. Douglas," Tommaso Caneva, and Darrick E. Chang \\ ICFO-Institut de Ciencies Fotoniques, The Barcelona Institute of Science and Technology, \\ 08860 Castelldefels, Barcelona, Spain
}

(Received 2 November 2015; published 4 August 2016)

\begin{abstract}
Realizing systems that support robust, controlled interactions between individual photons is an exciting frontier of nonlinear optics. To this end, one approach that has emerged recently is to leverage atomic interactions to create strong and spatially nonlocal interactions between photons. In particular, effective photonic interactions have been successfully created via interactions between atoms excited to Rydberg levels. Here, we investigate an alternative approach, in which atomic interactions arise via their common coupling to photonic crystal waveguides. This technique takes advantage of the ability to separately tailor the strength and range of interactions via the dispersion engineering of the structure itself, which can lead to qualitatively new types of phenomena. For example, much of the work on photon-photon interactions relies on the linear optical effect of electromagnetically induced transparency, in combination with the use of interactions to shift optical pulses into or out of the associated transparency window. Here, we identify a large new class of "correlated transparency windows," in which photonic states of a certain number and shape selectively propagate through the system. Through this technique, we show that molecular bound states of photon pairs can be created.
\end{abstract}

DOI: 10.1103/PhysRevX.6.031017

Subject Areas: Photonics, Quantum Physics

\section{INTRODUCTION}

In recent years, there has been tremendous progress to realize systems that are capable of achieving strong interactions between individual photons [1]. One common approach has been to couple single atoms (or other quantum emitters) to high-finesse optical cavities, to take advantage of the intrinsic nonlinear nature of these twolevel systems [2-8]. More recently, gases of cold Rydberg atoms have been investigated [9-15]. In this case, the optical nonlinearities are effectively generated via strong atom-atom interactions, with a novel consequence being that the nonlinearity becomes spatially nonlocal in character [16]. It has been experimentally shown that this type of nonlinearity can give rise to exotic states such as a twophoton bound state [17], and a number of other few- and many-body states of light have been theoretically predicted [18-20].

Key to this approach is that atoms excited to a Rydberg state, in particular, through the absorption of a photon, exert a strong dispersive effect on the level structure of proximal atoms, shifting their transition frequencies to Rydberg states by an amount proportional to $\sim 1 / r^{6}$, with $r$ being the interatomic separation [see Fig. 1(a)] [21]. This process,

\footnotetext{
*Corresponding author. james.douglas@icfo.eu

Published by the American Physical Society under the terms of the Creative Commons Attribution 3.0 License. Further distribution of this work must maintain attribution to the author(s) and the published article's title, journal citation, and DOI.
}

in turn, alters the optical susceptibility of the atoms, which can be interpreted as a change of refractive index that depends on the number and position of photons in the system. In practice, the $r$ dependence of the Rydberg interaction presents somewhat of a constraint. Specifically, one must reach extremely high Rydberg states in order to induce a significant nonlocal effect ( $n \sim 100$ in Ref. [17] to achieve a blockade radius of $18 \mu \mathrm{m})$. The resulting shift of the Rydberg level is large (about $50 \mathrm{GHz}$ for atoms separated by $4 \mu \mathrm{m}$ in Ref. [17]); however, all of the interesting variation in the atomic refractive index occurs over much smaller bandwidths of a few $\mathrm{MHz}$ (characteristic of the atomic linewidth). In this article, we demonstrate the new opportunities arising for nonlinear optics if the strength and range of the interaction can be independently adjusted. In particular, we show that the highly tunable nature of systems coupling atoms with nanophotonic devices can greatly extend the gamut of photon-photon interactions.

Our work is inspired by recent developments to interface cold atoms with photonic crystals [22-27]. It has been proposed that one can control the type and range of atomic interactions [28-32], and hence the interactions between photons [33], by engineering the underlying optical dispersion of these structures. We specifically study the dynamics of photons propagating through an atomic ensemble coupled to the waveguide under conditions of electromagnetically induced transparency (EIT), which occurs for atoms with three or more internal levels, such as shown in Figs. 1(a) and 1(b) [34]. Without long-range interactions, EIT is an interference effect that allows an 

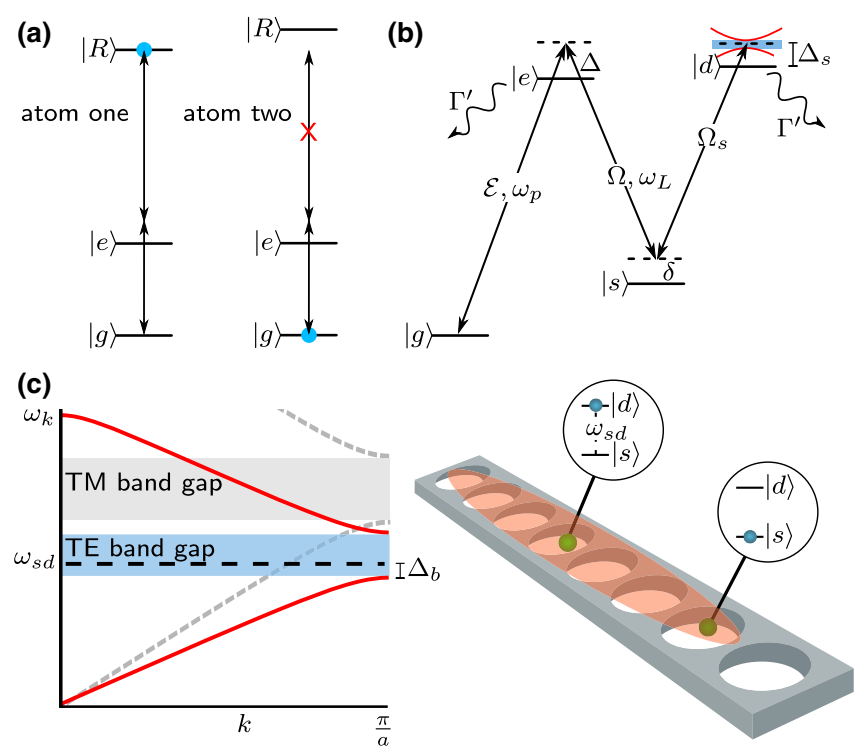

FIG. 1. (a) Rydberg interactions between atoms mediate photon-photon interactions. Two-photon excitation of atom 1 to Rydberg level $R$ shifts the $R$ level in other surrounding atoms by an amount much larger than the two-photon transition width, making the transition effectively two level for a second photon entering the system. (b) Four-level atom for EIT with interactions. The input quantum probe field $\mathcal{E}$ couples to the transition $|g\rangle-|e\rangle$, while transition $|s\rangle-|e\rangle$ couples to a classical drive field. Adding level $|d\rangle$ that couples to the band edge modes of a photonic crystal (red curves with blue region indicating the band gap) and driving the transition $|s\rangle-|d\rangle$ off-resonantly creates an effective interaction between atoms in state $|s\rangle$. For simplicity, we assume states $|e\rangle$ and $|d\rangle$ both have a free-space spontaneous decay rate $\Gamma^{\prime}$. (c) The periodic dielectric structure of a photonic crystal (right) leads to band structure (left) in the dispersion relation for photons propagating in the dielectric, where interference leads to band gaps over which propagation is forbidden. For photonic crystals that support multiple modes, e.g., TE and TM (represented here by the solid and dotted lines), the band gaps of different modes may occur at different frequencies. The $|s\rangle-|d\rangle$ transition of the atoms (green spheres) trapped near the photonic crystal couples to the photonic crystal bands. When the transition frequency $\omega_{s d}$ is in the band gap (with detuning $\Delta_{b}$ from the band edge) atoms can couple with one another via evanescent fields in the photonic crystal (illustrated in red), yielding a dipole-dipole interaction between the atoms.

optical probe field to propagate without absorption due to the interference created by a second pump field when a "two-photon resonance" condition is satisfied, $\delta=0$ in Fig. 1(b). As a linear optical effect, a probe pulse of any photon number and shape within a certain bandwidth propagates in this transparent manner.

Atomic interactions, for example, that shift level $|s\rangle$ in Fig. 1(b), shift the two-photon resonance, altering the propagation of photons in the system, and effective photon-photon interactions result. In all previous work, phenomena based upon on the incorporation of interactions into EIT only account for the possibilities of large interactions shifting a pulse out of the linear transparency window (e.g., photon blockade), or the interactions being small enough that the linear window still supports the multiphoton pulse. Here, we identify a remarkable new class of correlated transparency windows, which can arise when the interactions are sufficiently smooth and long range, in which only pulses of a desired photon number and shape will propagate through. As an example, we provide an explicit construction to generate a two-photon molecule consisting of two photons bound at a fixed separation by an effective spring, where phonon oscillations constitute the state's fundamental excitations.

The article is structured as follows. In Sec. II, we briefly review the physics governing atoms coupled to photonic crystal waveguides. In particular, we show how tunable long-range interactions between atoms emerge when the atomic transition frequency is situated within an optical band gap of the structure. In Sec. III, we show how EIT can be used to convert these atom-atom interactions into effective interactions between photons. In this regime, we find an effective equation for photons propagating in the system that supports the presence of correlated transparent states. In Sec. IV, we show how the photon interactions can be tuned to create molecularlike states of photons and, in Sec. V, we derive the conditions to observe such a state, demonstrating that the required parameters are within reach of state-of-the-art nanophotonic systems, before concluding in Sec. VI.

\section{INTERACTIONS MEDIATED BY PHOTONIC CRYSTALS}

Photonic crystals are dielectric structures in which the refractive index is modulated periodically [35]. At some frequencies, light input into the dielectric reflects constructively from these modulations, and the system acts like a mirror, preventing propagation. This leads to the presence of band gaps in the dispersion relation for the photonic modes, as shown in Fig. 1(c). When an atom trapped nearby the photonic crystal is excited at a frequency in the band gap, it is forbidden from emitting a propagating photon into the dielectric. However, it can generate an exponentially decaying evanescent field that forms a localized photonic cloud around the atom [36]. It has been formally shown that this photonic cloud has the same functionality as a real cavity of the same size [31], with an effective vacuum Rabi splitting $g_{c}$ dictated by the mode volume and an atom-cavity detuning $\Delta_{c}=2 \Delta_{b}$ set by the separation $\Delta_{b}$ between the atomic frequency and band edge. Thus, exactly as in a real cavity in the far-detuned regime $\left(\left|\Delta_{c}\right|>\left|g_{c}\right|\right)$ [37], atoms can exchange excitations via virtual "cavity" photons at a rate scaling with $g_{c}^{2} / \Delta_{c}$. In this case, the exchange is characterized by the dipole-dipole interaction Hamiltonian $H_{d d}=$ $\left[\hbar g_{c}^{2} / \Delta_{c}\right] \sum_{j, l} \sigma_{d s}^{j} \sigma_{s d}^{l} f\left(z_{j}, z_{l}\right)$, where $|s\rangle$ and $|d\rangle$ are the ground and excited states coupled by the cavity mode; see 
Fig. 1(c) [28-31,36,38-40]. Signatures of this interaction have recently been observed experimentally [27].

In the case where the atom is coupled predominantly to only a single band of a one-dimensional photonic crystal waveguide (achieved by tuning the atomic resonance close to the band edge), the interaction has spatial form $f\left(z_{j}, z_{l}\right)=\exp \left(-\left|z_{j}-z_{l}\right| / L\right) E_{k_{b}}^{*}\left(z_{j}\right) E_{k_{b}}\left(z_{l}\right)$ for atoms at positions $z_{j}$ and $z_{l}$, where $E_{k_{b}}(z)$ is the Bloch function of the photonic crystal at the band edge (wave vector $k_{b}$, frequency $\omega_{b}$ ). Below, we perform numerical simulations with the atoms trapped on a lattice with spacing $z_{a}=2 a$, for photonic crystal unit cell length $a$, in which case $E_{k_{b}}\left(z_{j}\right)=1$ at the trapping sites and the interaction is purely in the relative coordinate $\left|z_{j}-z_{l}\right|$. The interaction has length scale $L=\sqrt{\alpha \omega_{b} /\left(k_{b}^{2} \Delta_{b}\right)}$ corresponding to the length of the effective cavity mode, which depends on the detuning $\Delta_{b}$ as well as the curvature of the band edge $\alpha$. The resulting atom-atom interaction can be tuned in strength and length by adjusting these parameters, where a smaller detuning gives a longer-range interaction and flatter bands lead to a shorter-range interaction.

We wish to convert this dipole exchange interaction into a dispersive interaction between atoms in level $|s\rangle$, as required to shift the EIT two-photon resonance. To achieve this, we off-resonantly drive the $|s\rangle-|d\rangle$ transition of all atoms with Rabi frequency $\Omega_{s}$ and detuning $\Delta_{s}\left(\left|\Delta_{s}\right| \gg\left|\Omega_{s}\right|, g_{c}^{2} /\left|\Delta_{c}\right|\right)$, as shown in Fig. 1(b). In this case, virtual excitations of state $|d\rangle$ result in a Stark shift of level $|s\rangle$ for the $j$ th atom, which depends on the number of proximal atoms in state $|s\rangle$. After adiabatically eliminating the excited state $|d\rangle$, the interaction takes the general form given by the Hamiltonian $H_{s s}=-\hbar \sum_{j, l} \sigma_{s s}^{j} \sigma_{s s}^{l} V\left(z_{j}-z_{l}\right)$, where $\quad V\left(z_{j}-z_{l}\right)=$ - $\left[\left|\Omega_{s}\right|^{2} g_{c}^{2} /\left(\Delta_{s}^{2} \Delta_{c}\right)\right] f\left(z_{j}, z_{l}\right)$ for the single-band-edge coupling described above. For realistic experimental parameters, the coupling $g_{c}$ can be as large as $2 \pi \times 10 \mathrm{GHz}$ for a cavity that is just one wavelength long, $L \sim \lambda$ [31]. Importantly, the strength of the interaction can be tuned to any smaller value by altering the ratio $\left|\Omega_{s} / \Delta_{s}\right|$ for the driving laser, such that level shifts seen by the atoms can be on the order of the free-space linewidth $\gamma$, representing a significant distinction from the Rydberg case. The spatial dependence of the interaction can also be tuned; for example, exotic polynomial interactions such as $z^{-1 / 4}$ can be generated [31], or as we will show below, a molecular binding potential can be created.

The photonic-crystal-mediated interactions are not without losses. In a realistic system, imperfections in the dielectric medium cause loss of photons at rate $\kappa$. At the same time, the photonic crystals used in current experiments are not three dimensional, and the excited state $|d\rangle$ can spontaneously emit into free space at a rate $\Gamma^{\prime}$ comparable to $\gamma$. To understand the effect of these loss channels, we take advantage of the mapping of the photonic cloud surrounding the atom to an effective cavity mode. For an optimal choice of the detuning $\Delta_{b}$ from the band edge and the curvature $\alpha$ of the band, the two-atom dispersive interaction strength can be made larger than the residual dissipation by a factor $\sqrt{C} / 2$, where $C=g_{c}^{2} /\left(\kappa \Gamma^{\prime}\right)$ is the cooperativity of the cavity [31]. In state-of-the-art systems, the cooperativity can be as large as $C_{\lambda} \sim 10^{4}$ for a photonic cloud with an attenuation length $L$ equal to the wavelength $\lambda$. For longer range couplings with decay length $L$, the cooperativity scales as $C_{\lambda} \lambda / L$.

\section{EIT WITH INTERACTIONS}

The basis of EIT in an atomic medium is atoms with three internal levels $|g\rangle,|e\rangle$, and $|s\rangle$, as shown in Fig. 1(b). We consider a system where the atoms are coupled by the $|g\rangle-|e\rangle$ transition to a quantum probe beam $\mathcal{E}$ propagating in a one-dimensional waveguide, while the $|e\rangle-|s\rangle$ transition is coupled to an external control laser field (Rabi frequency $\Omega$ ), and the photonic-crystal-mediated interaction acts on level $|s\rangle$ as described above. An experimental system that allows for this configuration has been demonstrated in Refs. [23,24,26], where atoms are trapped near a photonic crystal waveguide. In this system, the band gaps for the transverse electric (TE) and transverse magnetic (TM) modes of the waveguide occur over different frequency ranges; see Fig. 1(c). This enables the probe field $\mathcal{E}$ to be guided in the TM mode and couple resonantly to the $|g\rangle-|e\rangle$ transition, while the long-range atom-atom interactions are mediated on the $|s\rangle-|d\rangle$ transition by the TE mode that has a band gap at the same frequency. At the same time, the control beam $\Omega$ may illuminate the atoms from free space.

With the control laser switched off $(\Omega=0)$, and atoms initialized in state $|g\rangle$, probe photons entering the atomic ensemble encounter a two-level medium. When the probe beam frequency $\omega_{p}$ is on resonance with the atomic transition $\left(\Delta=\omega_{p}-\omega_{g e}=0\right)$, the probe beam transmission is attenuated by a factor of $\exp (-D)$ after propagating through $N_{a}$ atoms, where $D=2 N_{a} \Gamma_{1 \mathrm{D}} / \Gamma^{\prime}$ is the optical depth (see Appendix A). Here, $\Gamma_{1 \mathrm{D}}$ denotes the emission rate of state $|e\rangle$ into the guided mode of the photonic crystal, while $\Gamma^{\prime}$ is the emission into all other modes (which in current experiments is comparable to the free-space emission rate $\gamma$ ), and $\Gamma=\Gamma_{1 \mathrm{D}}+\Gamma^{\prime}$ is the total single-atom linewidth [41].

Instead, when the control is switched on, the two-photon process of absorbing a probe photon and stimulated emission into the control field at frequency $\omega_{L}$ transfers atomic population to the internal $|s\rangle$ state from state $|g\rangle$. When this two-photon process is resonant, $\delta=\omega_{p}-\omega_{L}-$ $\omega_{g s}=0$ (in the absence of atom-atom interactions), interference between excitation from $|g\rangle$ to $|e\rangle$ and $|s\rangle$ to $|e\rangle$ leads to zero population in the excited state, and the medium becomes transparent as loss due to spontaneous 
emission is no longer possible [34]. In this case, the input probe photons are mapped to dark state polaritonssuperpositions of electromagnetic fields and spin coherence between the $|g\rangle$ and $|s\rangle$ states [42]. The presence of the atomic spin component allows the speed of propagation to be controlled by the external laser field $\Omega$, where the group velocity becomes $v_{g}=2|\Omega|^{2} /\left(\Gamma_{1 \mathrm{D}} n\right)$ for an atomic medium with linear density $n$ in the direction of propagation [43]. For realistic parameters, in the slow-light regime when $v_{g} \ll c$, the polariton excitations are almost completely spin wave in character; i.e., each polariton excitation effectively results in one atom being flipped to state $|s\rangle$. When atom-atom interactions are not present in the system, this transparency is a linear optical effect and applies for probe pulses of any photon number.

The situation changes dramatically when the photonic crystal mediated interactions $H_{s s}=-\hbar \sum_{j \neq l} \sigma_{s s}^{j} \sigma_{s s}^{l} V\left(z_{j}-\right.$ $z_{l}$ ) are added (note that we have excluded the diagonal level shift, $j=l$, from this Hamiltonian, which is assumed to be absorbed into the definition of our control laser frequency $\left.\omega_{L}\right)$. In particular, one polariton excitation results in a spatially dependent shift of level $|s\rangle$ for all surrounding atoms, as shown in Fig. 2(a). This implies that a second proximal polariton would generally not match the twophoton resonance condition, and its propagation is suppressed. Such an effect has already been exploited using Rydberg interactions to produce strong single-photon-level nonlinearities [9-15], where for Rydberg atoms the level shift is so large that the two-photon transition is no longer possible and the atom response is reduced to that of the two-level transition $|g\rangle-|e\rangle$. Using the photonic crystal interactions instead, where the strength and range of interaction associated with $H_{s s}$ can be tuned appropriately, novel phenomenon inaccessible with Rydberg atoms appear that have not been previously demonstrated. In particular, it is possible for a second polariton to propagate through, provided that its frequency and shape are specially matched to an altered two-photon resonance condition. Here, we identify and characterize the nature of this multitude of correlated transparency windows that depend both on the photon number and wave-packet shape, providing a powerful resource for the quantum manipulation of photonic states. A similar scheme for achieving photon-photon interactions is also described in Ref. [33], which however considers nonlinear phenomena that are distinct from the correlated transparent states considered here.

We first provide some intuitive examples of such behavior, before considering the physics in greater detail. We begin with the limit where the interaction is uniform over the atomic sample, which could be achieved by tuning the interaction length $L$ to be much larger than the size of the atomic ensemble, so that $V\left(z_{j}-z_{l}\right) \sim-U$. In this case, the interactions lead to energy shifts that are independent of where the polaritons are [43]. A single photon propagating

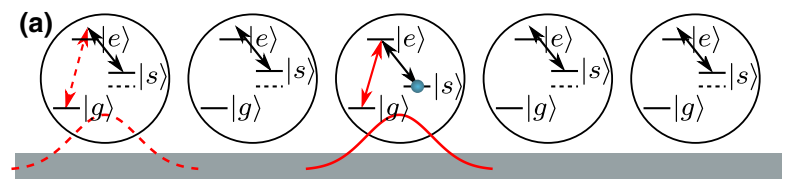

(b)
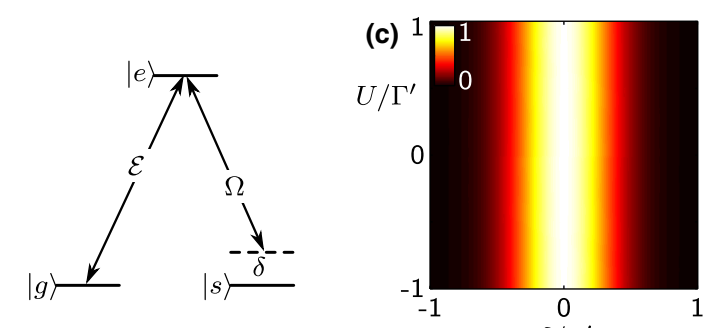

(d)

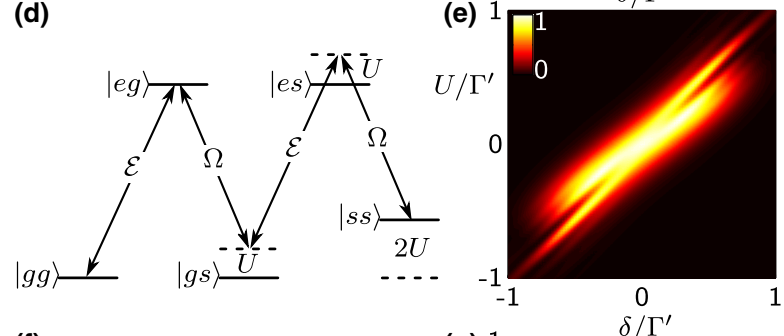

(f)

(g)

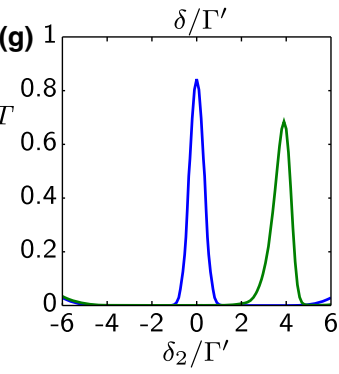

FIG. 2. (a) The excitation of the central atom to $|s\rangle$ by a probe pulse (red line) traveling along the waveguide (grey), shifts the level $|s\rangle$ of the surrounding atoms and a new pulse (dashed red line) encounters a shifted EIT transparency window. (b-e) EIT with uniform interactions $H_{s s}=\hbar U \sum_{j \neq l} \sigma_{s s}^{j} \sigma_{s s}^{l}$ leads to numbercorrelated transparency windows. (b) For a single photon, EIT occurs at the normal two-photon resonance $\delta=0$, independently of $U$, as shown in (c) by the simulated output intensity $I_{1}=\left\langle\mathcal{E}_{\mathrm{o}}^{\dagger} \mathcal{E}_{\mathrm{o}}\right\rangle /\left\langle\mathcal{E}_{\mathrm{i}}^{\dagger} \mathcal{E}_{\mathrm{i}}\right\rangle$, which for weak input intensity corresponds to the transmittance of single photons. (d) For two photons, EIT occurs for $\delta=U$, in which case transfer to the $|s s\rangle$ two-excitation state is resonant, as shown in (e) by the equal-time coincidence rate at output $I_{2}=\left\langle\mathcal{E}_{\mathrm{o}}^{\dagger} \mathcal{E}_{\mathrm{o}}^{\dagger} \mathcal{E}_{\mathrm{o}} \mathcal{E}_{\mathrm{o}}\right\rangle /\left\langle\mathcal{E}_{\mathrm{i}}^{\dagger} \mathcal{E}_{\mathrm{i}}\right\rangle^{2}$, which has a maximum for $\delta \sim U$. In (c) and (e), the system has optical depth $D=400$ and is continuously pumped with $\left|\mathcal{E}_{\mathrm{i}}\right|^{2}=10^{-4} \Gamma^{\prime}$ and $\Delta=0$. (Note that $\delta=U$ can be achieved by adjusting the probe or control field frequencies; here, we adjust the control, which in this case results in higher two-photon coincidence counts for $|U|>0.5$.) (f) Twophoton transmission is also achieved by inputting two pulses with different frequencies $\omega_{1}$ and $\omega_{2}$, where the first pulse is inside the medium when the second arrives [as in (a)]. For $\delta_{1} \sim 0$, a single photon from the first pulse may propagate in the medium, while, taking $\delta_{2} \sim 2 U$, a photon from the second pulse may only enter when a photon from the first is inside the medium. (g) The presence of the first photon then switches the probability of transmission $T$ for the second photon from being maximized at $\delta_{2}=0$ (blue curve) to $\delta_{2}=2 U$ (green curve), with $U=2 \Gamma^{\prime}$. All simulations were done with $\Omega=2 \Gamma^{\prime}, \Gamma_{1 \mathrm{D}}=2 \Gamma^{\prime}$, and $N_{a}=100$. 
by itself in such a medium will be maximally transmitted through the atomic medium when $\delta=0$ [Fig. 2(b)], while for two photons to enter the system, their detunings must compensate for the shift to the doubly excited state $|s s\rangle$. This could be achieved as shown in Fig. 2(d) with an input such that $\delta=U$. More generally, we have numbercorrelated transparency windows, where $N_{p}$ photons will be transmitted maximally when $\delta=\left(N_{p}-1\right) U$. Significantly, these transparency windows exist simultaneously in the system, and there is no requirement for the transparency windows associated with different $N_{p}$ to overlap with one another.

This type of effect could not be realized with pure Rydberg interactions, even if the Rydberg blockade were extended across the entire sample, because of their rapid $1 / r^{6}$ spatial variation. In general, correlated transparency windows are difficult to access for such a potential as the spatial region over which the level shift due to the interaction fits within the EIT transparency is limited. For example, for the interatomic potential used in Ref. [17], the level shift changes from $2 \gamma$ to $3 \gamma$ over a distance of $0.9 \mu \mathrm{m}$. Such small spatial regions in turn lack sufficient optical depth in which to compress a pulse, at least for atomic densities used in current experiments, and resulting pulse propagation would be highly lossy.

In Figs. 2(c) and 2(e), we plot measures of single- and two-photon output from an EIT medium given an input of continuous coherent light over a range of two-photon detunings $\delta$ and uniform interaction strengths $U$. The output probe field $\mathcal{E}_{\mathrm{o}}$ is produced numerically for a particular input $\mathcal{E}_{\mathrm{i}}$ by simulating the full EIT system using the spin model from Ref. [43], which we further describe below. We plot the normalized output intensity $I_{1}=\left\langle\mathcal{E}_{\mathrm{o}}^{\dagger} \mathcal{E}_{\mathrm{o}}\right\rangle /\left\langle\mathcal{E}_{\mathrm{i}}^{\dagger} \mathcal{E}_{\mathrm{i}}\right\rangle$, which for weak input probe intensity corresponds to the transmission of single photons through the system. $I_{1}$ peaks at the normal EIT resonance $\delta=0$ and is independent of the interaction strength. To visualize the two-photon behavior, we plot the normalized two-photon coincidence $I_{2}=\left\langle\mathcal{E}_{\mathrm{o}}^{\dagger} \mathcal{E}_{\mathrm{o}}^{\dagger} \mathcal{E}_{\mathrm{o}} \mathcal{E}_{\mathrm{o}}\right\rangle /\left\langle\mathcal{E}_{\mathrm{i}}^{\dagger} \mathcal{E}_{\mathrm{i}}\right\rangle^{2}$, which is proportional to the rate at which two photons are detected leaving the system at the same time. $I_{2}$ peaks when the two-photon transmission is resonant, which occurs when $\delta \sim U$ and confirms the intuition presented earlier. The deviation from unit two-photon transmission for larger values of $U$ $\left(|U| \gtrsim \Gamma^{\prime}\right)$ is due to the fact that for a continuous wave input, the two photons are entering and leaving at random times. Thus, each photon partially experiences the loss associated with the single-photon dispersion relation at a frequency displaced from the transparency condition [43].

In the previous example, we probed the two-photon transparency using a constant, coherent input with one frequency as shown in Fig. 2(d). An alternative way to drive the two-photon transparency is with two individual coherent pulses of different frequencies $\omega_{1}$ and $\omega_{2}$ as shown in Fig. 2(f). The frequency of the first pulse is arranged to satisfy the single-photon transparency so that $\delta_{1}=\omega_{1}-\omega_{L}-\omega_{g s} \sim 0$, in which case single photons from this pulse propagate transparently through the medium, while higher-number components are dissipated because of the uniform interactions. The second pulse is arranged to enter the medium when the first is already completely inside as shown schematically in Fig. 2(a), with a shifted frequency to achieve two-photon resonance so that $\delta_{2} \sim \delta_{1}+\delta_{2} \sim 2 U$. In this case, propagation of the photons from the second pulse is conditional on a photon from the first pulse already being inside the medium. Given the presence of a single photon from the first pulse, one and only one photon from the second pulse may propagate through the medium; otherwise, the second pulse is dissipated completely. In other words, the first pulse acts like a switch for the second pulse. We show this behavior in Fig. 2(g), where we have plotted the simulated transmission probability of a single photon from the second pulse with and without the presence of a photon from the first as we vary $\delta_{2}$. Here, we see that the presence of the first photon shifts the transparency window for the second pulse from $\delta_{2} \sim 0$ to $\delta_{2} \sim 2 U$ and that these windows do not overlap, allowing the first pulse to switch the transmission of a photon in the second pulse on or off as predicted.

An extension of the example of uniform interactions is to consider a step potential, where the interaction is a constant $U$ up to a certain atom separation $r_{s}$ and then zero at larger distances, as shown in Fig. 3(a). This situation leads to the presence of spatial- and number-correlated transparent states of photons, where two photons may propagate together with separation $r<r_{s}$ for an input such that $\delta \sim U$, or separated with $r>r_{s}$ when $\delta \sim 0$. Since the darkstate polariton excitations essentially consist of atoms excited to state $|s\rangle$, we can visualize this behavior by plotting the probability $\left|\psi_{s s}^{j, l}\right|^{2}$ of having two atoms $(j, l)$ both excited to $|s\rangle$. In Fig. 3(b), we plot this quantity for $\delta=0$ in a steady state when the system is driven by a weak continuous probe input. In this case, the step potential leads to minimal population of polaritons separated by less than $r_{s}$. On the other hand, when $\delta \sim U$, we expect the polaritons to propagate together within the step separation $r_{s}$. In fact, one expects that if the wave function of the relative coordinate of a two-photon state is prepared as a harmonic eigenmode of the square-well potential [Fig. 3(a)], it can propagate, maintaining its pulse shape in that direction. This is illustrated in Fig. 3(c) for the case where the relative wave function is initially prepared in the third harmonic. The relative coordinate maintains its shape well, while the wave function in the center-of-mass coordinate diffuses out.

Motivated by these basic intuitive and numerical observations, we seek to better understand the dynamics of photons within spatial- and frequency-correlated transparency windows. To this end, a more rigorous description of the EIT system can be developed by finding the effective Hamiltonian for the polariton dynamics in the presence of 

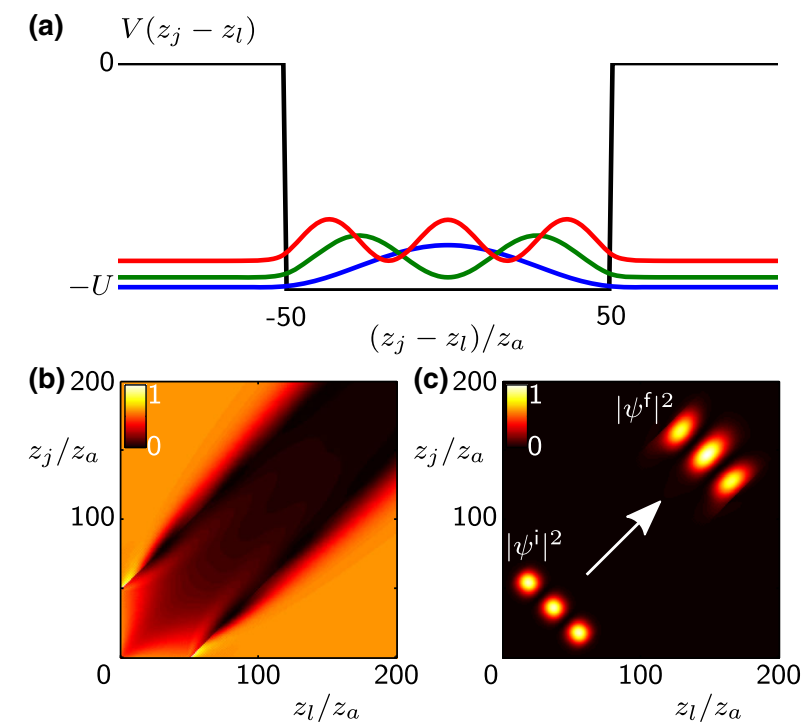

FIG. 3. EIT in atomic medium with $N_{a}=200$ atoms on a lattice with lattice constant $z_{a}$ and interaction $H_{s s}=$ $-\hbar \sum_{j \neq l} \sigma_{s s}^{j} \sigma_{s s}^{l} V\left(z_{j}-z_{l}\right)$. (a) The potential is chosen to be a square well, with an interaction strength of $V\left(z_{j}-z_{l}\right)=-0.5 \Gamma^{\prime}$ for atoms with separation $\left|z_{j}-z_{l}\right|<50 z_{a}$ and zero otherwise. The colored curves show the three lowest energy states of the step potential. (b) When the system is driven continuously so that $\delta=0\left(\left|\mathcal{E}_{\mathrm{i}}\right|^{2}=10^{-4} \Gamma^{\prime}\right)$, the majority of the steady-state spin population $\left|\psi_{s s}^{j, l}\right|^{2}$ (arbitrary units) is in pairs of excitations with $\left|z_{j}-z_{l}\right|>50 z_{a}$. (c) Propagation of a two-photon wave function (arbitrary units) in which the relative coordinate is initially prepared as the third harmonic eigenstate [red curve in (a)] of the square-well potential. Here, the final spin population is multiplied by a factor of 100 , so both initial $\left|\psi^{i}\right|^{2}$ and final $\left|\psi^{f}\right|^{2}$ are visible on the same scale. Other simulation parameters used are $\Omega=2 \Gamma^{\prime}, \Gamma_{1 \mathrm{D}}=\Gamma^{\prime}$ (optical depth $D=400$ ), and $\Delta=0$.

the atom-atom interactions. By perturbatively solving the Heisenberg equations of motion around points where such correlated windows are created (as described in Appendix B), we find

$$
\begin{aligned}
H_{\mathrm{eff}}= & \hbar \int d z \Psi^{\dagger}(z)\left[-i v_{g} \frac{\partial}{\partial z}-\frac{\hbar}{2 m} \frac{\partial^{2}}{\partial z^{2}}\right. \\
& \left.-\delta-\int d z^{\prime} \Psi^{\dagger}\left(z^{\prime}\right) V\left(z-z^{\prime}\right) \Psi\left(z^{\prime}\right)\right] \Psi(z),
\end{aligned}
$$

for the dark-state polariton field operator $\Psi(z)$.

This effective Hamiltonian highlights a number of features of polariton evolution, the first being the wellknown reduced group velocity, $v_{g} \ll c$, of the polaritons in the medium, seen here as the first term in the brackets. Meanwhile, the interaction between the polaritons that results from the atom-atom interactions is described by the final term in the brackets. Here, the interaction itself $V\left(z-z^{\prime}\right)$ does not need to be small or correspond to a level shift within the normal EIT transparency bandwidth (e.g., as assumed in Ref. [18]). Instead, we require that the change in the interaction over the region where there is significant polariton density lies within the transparency bandwidth, and that for an $N_{p}$ photon transparency, the detuning of the input photons compensates for the interaction energy when $N_{p}$ polaritons are in the medium, i.e., $N_{p} \delta+\int d z d z^{\prime} \Psi^{\dagger}(z) \Psi^{\dagger}\left(z^{\prime}\right) V\left(z-z^{\prime}\right) \Psi\left(z^{\prime}\right) \Psi(z) \sim 0$. In particular, Eq. (1) can describe different self-consistent transparency windows, for different photon number and spatial configurations, and these need not overlap.

The second term in brackets describes the dispersion of the group velocity, which is equivalent to the polariton having an effective mass in the EIT medium $m=-\hbar|\Omega|^{2} /\left[\left(2 \Delta_{M}+i \Gamma^{\prime}\right) v_{g}^{2}\right]$. The effective mass is a complex parameter with a real part depending on a renormalized probe detuning $\Delta_{M}$ and the imaginary part on the spontaneous decay rate into other modes $\Gamma^{\prime}$. The renormalization of the detuning, $\Delta_{M}=\Delta+\delta$, results from the different bare two-photon detuning $\delta$ required to achieve EIT transparency in the interacting system. In general, the mass depends on the number of polaritons in the system [33], for example, in the case of uniform interactions where $\delta$ depends on $N_{p}$ (see Appendix B for further details).

For two photons, Eq. (1) separates into center-of-mass and relative coordinates, where each coordinate has a mass or diffusion term exactly like in noninteracting EIT. In particular, a well-localized wave function experiences a similar loss as in normal EIT. However, the relative coordinate also sees the effective potential, which could, for example, support bound states as we describe in the next section. In general, we can map a multipolariton system to a collection of massive particles in a potential moving in a reference frame traveling at $v_{g}$.

The effective Hamiltonian of Eq. (1) is valid around the two-photon resonance, where we expect it to provide an approximate description of the full system dynamics. To confirm these dynamics, we numerically simulate the full system using the spin model introduced in Ref. [43]. The spin model describes the behavior of many atoms coupled to the photonic modes of a one-dimensional waveguide, generalizing the powerful input-output formalism of cavity QED. The key insight of the model is that the exchange of photons (with wave vector $k_{p}$ ) between atoms via the waveguide reduces to an effective interaction between the atoms, given by

$$
H_{\mathrm{wg}}=-\frac{i \hbar \Gamma_{1 \mathrm{D}}}{2} \sum_{j, l} \sigma_{e g}^{j} \sigma_{g e}^{l} e^{i k_{p}\left|z_{j}-z_{l}\right|} .
$$

The dynamics of the electromagnetic field as it propagates through the system are now completely captured in the dynamics of the interacting spin chain with Hamiltonian 
$H_{\text {spin }}=H_{\text {wg }}+H_{\text {atom }}+H_{\text {pump }}+H_{s s}, \quad$ where $\quad H_{\text {atom }}$ describes the atomic Hamiltonian in the absence of the waveguide-mediated interactions and $H_{\text {pump }}$ describes the coupling of the atoms to input light. Below, we consider the case where the input probe light is coherent, in which case the coupling to the atoms can be accounted for by using the Mollow transformation [44], and we have $H_{\text {pump }}=-\hbar \mathcal{E}_{\mathrm{i}}(t) \sqrt{\left(\Gamma_{1 \mathrm{D}} / 2\right)} \sum_{j}\left(\sigma_{e g}^{j} e^{i k_{p} z_{j}}+\sigma_{g e}^{j} e^{-i k_{p} z_{j}}\right)$. Here, $\mathcal{E}_{\mathrm{i}}(t)$ is a classical field that may either be constant, corresponding to continuous driving of the system, pulsed, or zero when we initialize the system with a spin wave.

The output field operator $\mathcal{E}_{\mathrm{o}}(z, t)=\mathcal{E}_{\mathrm{i}}(t-z / c)+$ $\varepsilon_{\mathrm{i}}(t-z / c)+i \sqrt{\left(\Gamma_{1 \mathrm{D}} / 2\right)} \sum_{j=1}^{N} \sigma_{g e}^{j}(t) e^{i k_{p}\left(z-z_{j}\right)}$ is determined from the coherent input, with classical part $\mathcal{E}_{\mathrm{i}}$ and vacuum fluctuations $\varepsilon_{\mathrm{i}}$, and the atomic coherence $\sigma_{g e}^{j}$ found by solving the spin evolution. The numerical description of the system is then reduced to following the dynamics of the discrete atomic Hilbert space under $H_{\text {spin }}$ instead of the inprinciple continuous electromagnetic field. This description allows us to fully model our EIT system, including arbitrary interactions between the atoms, in a way that is independent of the assumptions made in deriving the effective polariton dynamics in Eq. (1), providing numerical verification of the intuitive picture of polaritons as massive interacting particles.

\section{PHOTONIC MOLECULES}

We now use our effective propagation equation along with the spin model to describe a regime where photons can bind to one another in the EIT medium. Here, we rely on the tunability of the interactions in the photonic crystal setting to achieve a molecularlike interaction potential between polaritons. In particular, we consider the case where the $|s\rangle-|d\rangle$ transition couples in the band gap to both band edges, as shown in Fig. 1(b). In this case, the resulting effective interaction $V\left(z_{j}-z_{l}\right)=-\left[\left|\Omega_{s}\right|^{2} / \Delta_{s}^{2}\right]\left[\left(g_{u}^{2} / \Delta_{u}\right) \exp \left(-\left|z_{j}-z_{l}\right| / L_{u}\right)+\right.$ $\left.\left(g_{\ell}^{2} / \Delta_{\ell}\right) \exp \left(-\left|z_{j}-z_{l}\right| / L_{\ell}\right)\right]$ will be the sum of the interaction due to the upper $(u)$ and lower $(\ell)$ band edges (see Appendix C). Crucially, the sign of each contribution depends on the detuning from the band edge in question, and the contributions hence have opposite signs. Furthermore, by engineering the detunings and band curvatures correctly, the contributions will also have different interaction length scales, leading the total interaction to have a minimum (or maximum) at some atomic separation $r_{0}$, as plotted in Fig. 4(a) for the case where the interaction strengths have equal magnitude $G \equiv\left|\Omega_{s}\right|^{2} g_{u}^{2} /\left(\Delta_{s}^{2} \Delta_{u}\right)=\left|\Omega_{s}\right|^{2} g_{\ell}^{2} /\left(\Delta_{s}^{2} \Delta_{\ell}\right)$. We note that while the detunings $\Delta_{u}$ and $\Delta_{\ell}$ should be of the order of $100 \mathrm{GHz}$ to create the interaction, the gap between the band edges can be arbitrary if instead of a single excited level $|d\rangle$ we use one for each band edge. This
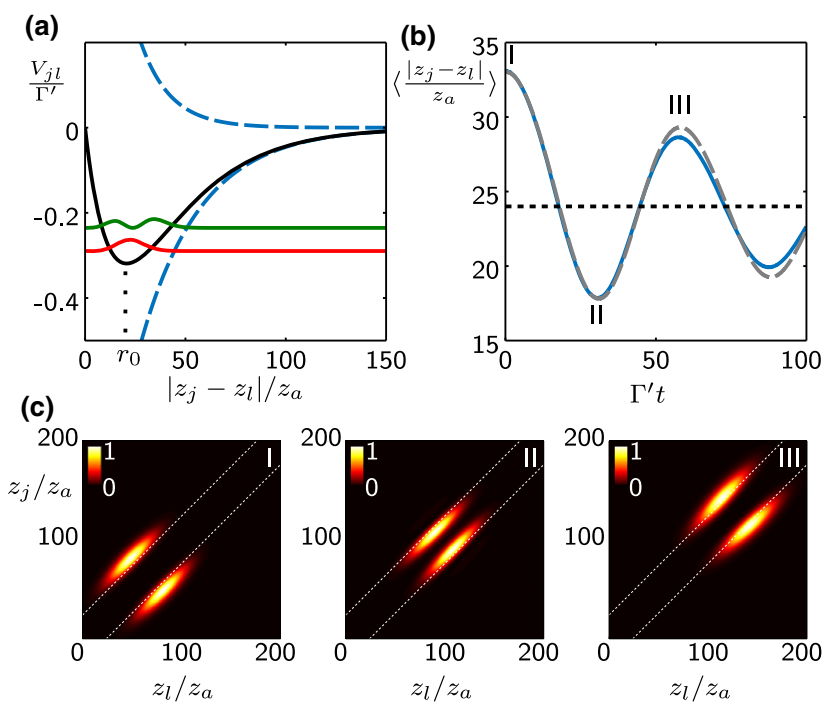

FIG. 4. (a) The photonic-crystal-mediated atomic interactions result in an interaction potential $V_{j l}=V\left(z_{j}-z_{l}\right)=$ $G\left[\exp \left(-\left|z_{j}-z_{l}\right| / L_{u}\right)-\exp \left(-\left|z_{j}-z_{l}\right| / L_{\ell}\right)\right]$ (black solid line) for polaritons separated by distance $\left|z_{j}-z_{l}\right|$. The interaction potential is the sum of the contribution from the lower and upper photonic crystal band edges (dashed blue lines) that have length scales $L_{u}=15 z_{a}$ and $L_{\ell}=30 z_{a}$ and strength $G=1.28 \Gamma^{\prime}$, which in this case leads to a minimum at a finite atomic separation $r_{0} \sim 21 z_{a}$. Loss resulting from the photonic-crystal-mediated interactions is calculated assuming a cooperativity of $C_{\lambda}=24000$. The amplitudes $|\psi(r)|^{2}$ (arbitrary units) of the two lowest bound-state wave functions are shown for $\Delta_{M}=2.5 \Gamma^{\prime}, \Omega=\Gamma^{\prime}$, and $\Gamma_{1 \mathrm{D}}=2 \Gamma^{\prime}$. (b) For two polaritons propagating with this interaction, a state initiated with a wave function matching that of the ground state, but offset from the ground-state separation at time $t=0$, oscillates in time in the relative coordinate. The oscillation of the expectation value of polariton separation about the ground-state separation (about $24 z_{a}$, indicated by the dotted line) found by simulation the full EIT system (solid line) is closely matched to the oscillation produced by the evolution of the effective Hamiltonian $H_{\text {rel }}=$ $-\left(\hbar^{2} / m\right)\left(\partial^{2} / \partial r^{2}\right)-2 \hbar V(r)$ (dashed line). In this case, a full oscillation occurs after the polaritons travel an optical depth of $D=250$ using the parameters in (a). (c) Spin population $\left|\psi_{j, l}^{s s}\right|^{2}$ (arbitrary units) as the initially offset pair of polaritons propagate through the atomic ensemble, with the dotted line indicating the ground-state separation of the two polaritons. The frames I, II, and III correspond to the turning points of the oscillation marked in (b).

configuration would coincide with current experiments where the D1 and D2 lines of cesium are aligned with the lower and upper band edges, respectively [23].

For the case of two polaritons, with coordinates $z_{1}$ and $z_{2}$, Eq. (1) separates into a Hamiltonian $H_{\mathrm{cm}}=$ $-\left[\hbar^{2} /(4 m)\right]\left(\partial^{2} / \partial R^{2}\right)-i \hbar v_{g}(\partial / \partial R)$ for the center-ofmass coordinate $R=\left(z_{1}+z_{2}\right) / 2$, whose dispersion corresponds to that of a free massive particle, and a Hamiltonian $H_{\text {rel }}=-\left(\hbar^{2} / m\right)\left(\partial^{2} / \partial r^{2}\right)-2 \hbar V(r)$ for the 
relative coordinate $r=z_{1}-z_{2}$. From $H_{\text {rel }}$, we can find the bound states of pairs of polaritons in the system, as shown in Fig. 4(a). An interesting bound state with finite separation is also predicted in Rydberg systems [20]. However, as Rydberg systems are not compatible with correlated transparency windows, such a bound state involves an admixture of dark and bright polaritons and thus suffers from increased dissipation. More generally in Rydberg systems, bound states arise because of the presence of sharp changes in the refractive index of the medium [17]. Instead, our system allows for a harmoniclike interaction, allowing fundamental phononic oscillations of the polaritons to be observed. In this way, our system mimics molecules formed by atoms.

In particular, initializing the two-polariton wave function in the motional ground state of the potential shown in Fig. 4(a) leads to the two polaritons propagating through the system with fixed relative position, while perturbing the state by offsetting the initial relative position results in molecular vibrations about this ground-state separation (slightly offset from $r_{0}$ because of the asymmetry of the potential). In Fig. 4(b), we plot the decaying oscillation of the relative position about the ground-state separation for a full spin-model simulation of a two-polariton wave function that has an initial offset, and we see good agreement with the oscillation predicted by the simple model. In Fig. 4(c), the polariton wave packet in the two excitation manifold is plotted at the extrema of the oscillations, where we see that the relative coordinate remains tightly bound, while the wave function in the center-of-mass coordinate disperses as a free massive particle.

In an experiment, the photon molecules could be observed using spin-dependent imaging of the cold atoms; however, the most straightforward measurements are of photons output from the system into the waveguide. Using the spin model, we can simulate an experiment where a weak coherent pulse is input into the system at a frequency resonant with the two-photon molecule $\left[\delta \sim-V\left(r_{0}\right)\right]$ and the output fields are recorded after the photons are allowed to propagate through the system. In Fig. 5(a) and 5(b), we plot the single- and two-photon parts of the input and output fields for a weak pulse with a Gaussian spatial envelope. Here, we see that the correlated transparency window damps most of the two-photon component of the initial Gaussian input, and what remains at the output largely coincides with the well-separated two-photon molecule. This result leads to a peak in the second-order correlation function $g^{(2)}(\tau)=\left\langle\mathcal{E}_{\mathrm{o}}^{\dagger}(t) \mathcal{E}_{\mathrm{o}}^{\dagger}(t+\tau) \mathcal{E}_{\mathrm{o}}(t+\tau) \mathcal{E}_{\mathrm{o}}(t)\right\rangle /$ $\left(\left\langle\mathcal{E}_{\mathrm{o}}^{\dagger}(t) \mathcal{E}_{\mathrm{o}}(t)\right\rangle\left\langle\mathcal{E}_{\mathrm{o}}^{\dagger}(t+\tau) \mathcal{E}_{\mathrm{o}}(t+\tau)\right\rangle\right)$ for the photons, Fig. 5(c), which shows large bunching due to the presence of the bound photon pair.

We can also observe the phononic oscillations of the pair of photons in the photon output. Inputting photons into the system in a Gaussian pulse as above, the photons are initially separated, on average, by less than the ground-state (a)

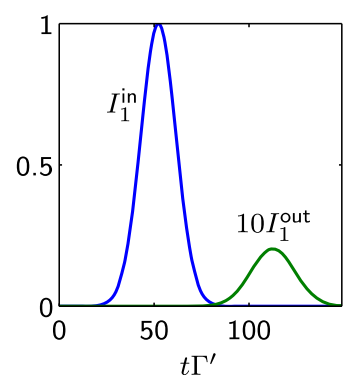

(c)

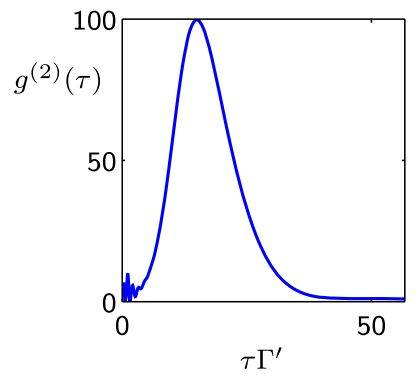

(d)
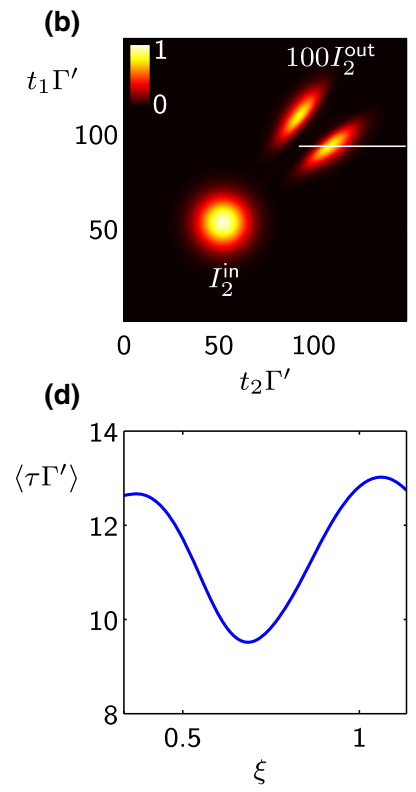

FIG. 5. Input and output of an EIT system with molecularlike interactions resulting from the potential shown in Fig. 4(a). (a) Single-photon component of input (blue line) and delayed output (green line) pulses, normalized by the peak intensity of the input pulse, where the output has been multiplied by a factor of 10 for visibility. (b) Two-photon input and output components, where the output has been scaled by a factor of $10^{2}$. (c) Secondorder correlation function $g^{(2)}(\tau)$ for output two-photon separation time $\tau=\left|t_{1}-t_{2}\right|$ taken along the white line shown in (b). (d) Oscillation in average two-photon separation time $\langle\tau\rangle$ at output as the interaction strength and renormalized detuning are scaled to become $G^{\prime}=\xi G$ and $\Delta_{M}^{\prime}=\xi \Delta_{M}$. The optical depth is 400 for plots (a)-(c) and 800 for plot (d), with $G=1.28 \Gamma^{\prime}$, $\Delta_{M}=1.5 \Gamma^{\prime}, \Omega=1.5 \Gamma^{\prime},\left|\mathcal{E}_{\mathrm{i}}\right|^{2}=10^{-4} \Gamma^{\prime}$, and $\Gamma_{1 \mathrm{D}}=2 \Gamma^{\prime}$.

separation, which initializes an oscillation in the twophoton wave function. The relative position of the two photons at the output depends on the ratio of the length over which the pulse travels in one oscillation period, $L_{o}$, to the length of the atomic medium, $L_{s}$. An oscillation can then be observed at output by either adjusting the effective length of the system, for example, by reducing the number of atoms by waiting for the atomic trap to decay, or by adjusting the strength $G$ of the interaction potential, for example, by adjusting the drive Rabi frequency $\Omega_{s}$. In the latter case, the oscillation length is proportional to $1 / \sqrt{\Delta_{M} G}$. However, adjusting the strength $G$ alone also changes the shape of the molecule, as the spatial extent of the ground state is approximately proportional to $\sqrt{\Delta_{M} / G}$. Reducing both $\Delta_{M}$ and $G$ by the same factor $\xi$ then results in the molecule keeping its shape while increasing $L_{\mathrm{o}}$ by a factor of $1 / \xi$. In Fig. 5(d), we show a simulation of this situation, where we adjust $G$ and $\Delta_{M}$ from the values used in Figs. 5(a)-5(c) that initially lead to approximately oneand-a-half oscillations over the length of the system. By reducing the values by a factor of 3 , the exact simulation 
shows a full oscillation in the average time separation of the two photons at output; i.e., $L_{\mathrm{o}}$ changes from $3 / 2 L_{s}$ to $1 / 2 L_{s}$, confirming the prediction of our simple model.

\section{TECHNICAL REQUIREMENTS}

Having described the main features of the two-photon molecule in the previous section, we now analyze, more carefully, the technical requirements in order to observe this physics. There are two main considerations, the first being the regime of validity of the effective equation (1) that describes polaritons as massive particles propagating in a potential, and the second, the extent to which dissipation affects the molecular state. Describing the polariton dynamics by Eq. (1) is equivalent to keeping only terms of up to quadratic order in detuning in the expansion of the photon dispersion relation about the EIT resonance. Neglecting higher-order terms is a good approximation within the EIT window, which for $|\Delta|>|\Omega|, \Gamma^{\prime}$, requires that the spread of frequencies of the relevant system dynamics remains within $|\Omega|^{2} /|\Delta|$ of the EIT resonance. For a pulse with length $z_{0}$ within the atomic medium [e.g., a Gaussian intensity distribution $\left.I(z) \propto \exp \left(-8 z^{2} / z_{0}^{2}\right)\right]$ to be described by Eq. (1), it must have frequency width $d \omega \sim 4 v_{g} / z_{0}<|\Omega|^{2} /|\Delta|$, which can be recast as a requirement $D_{p}>16|\Delta| / \Gamma^{\prime}$ for the optical depth $D_{p}$ in the pulse length. This identifies a trade-off, where we would like to increase the ratio $|\Delta| / \Gamma^{\prime}$ to reduce the effect of dissipation in the effective mass term; however, to do so requires an increase in the optical depth needed in an experiment.

Dissipation in our system comes from two main sources, one being spontaneous emission from the excited state $|e\rangle$ that gives rise to the imaginary part of the effective mass term, and the other being the additional loss associated with introducing the photonic-crystal-mediated interactions. The former leads to loss as a pulse propagates over length $L$ in the atomic medium, which in the limit of validity of the quadratic dispersion is approximately $\exp \left[-16 L /\left(z_{0} D_{p}\right)\right]$. This is the loss for linear propagation of a pulse, and for two photons propagating in a molecule, the loss is squared. To observe an oscillation of a photonic molecule, we would then like to take a minimal value of the oscillation length $L_{o}$ to reduce loss. To see how $L_{o}$ is constrained, we consider the case where the interaction potential can be approximately described as a harmonic oscillator, with Gaussian ground-state wave function $\exp \left[-2\left(r-r_{0}\right)^{2} /\left(z_{0}^{2}\right)\right]$ in relative coordinate space, in which case the photon molecule would oscillate with frequency $\omega_{M} \approx 32 v_{g}|\Delta| /\left(\Gamma_{1 \mathrm{D}} n z_{0}^{2}\right)$. The oscillation length $L_{o}=2 \pi v_{g} / \omega_{M}$ is reduced by increasing $\omega_{M}$; however, this frequency is constrained to remain within the EIT window, which then results in the restriction $L_{o}>\pi z_{0} / 2$. Taking the minimum value for $L_{o}$ and equating this with the system length $L_{s}$, such that the photon molecule undergoes one full oscillation, then yields a propagation loss of $\exp \left[-16 \pi / D_{p}\right]$.
As noted in Sec. II, the loss related with the photoniccrystal-mediated interactions depends on the cooperativity of the atom-induced cavities in the photonic crystal. For a potential like that in Fig. 4(a), the loss rate is approximately $\beta \omega_{M} \sqrt{z_{0} /\left(\lambda C_{\lambda}\right)}$ (taking $r_{0} \sim z_{0}$ ), where the proportionality constant $\beta$ depends on the details of how the two potentials are combined (see Appendix C). Over one oscillation period of the photonic molecule, we then expect the norm of the two-photon wave function to be reduced by a factor of approximately $\exp \left[-\left(2 \pi \beta \sqrt{z_{0}}\right) / \sqrt{\lambda C_{\lambda}}\right]$ as a result of this loss. Compared with the propagation loss, this loss increases with increasing pulse length $z_{0}$, as larger constituent pulses require larger effective cavities to create the potential in the photonic crystal. The total loss then represents a balance between the two contributions, and an optimal pulse length can be found, $z_{0}=$ $2\left[4 C_{\lambda} \Gamma^{2} /\left(\beta \Gamma_{1 \mathrm{D}}\right)^{2}\right]^{1 / 3}$, at which point the total loss is $\exp \left\{-6 \pi\left[2 \beta^{2} \Gamma^{\prime} /\left(C_{\lambda} \Gamma_{1 \mathrm{D}}\right)\right]^{1 / 3}\right\}=\exp \left(-48 \pi / D_{p}\right)$. For parameters compatible with current experimental setups, $\Gamma_{1 \mathrm{D}}=\Gamma^{\prime}, \beta \sim 10, z_{a} \sim \lambda$, and $C_{\lambda}=2 \times 10^{4}$, we have $z_{0} \sim$ $20 z_{a}$ and the $\exp \left(-48 \pi / D_{p}\right) \sim 0.02$. The total optical depth to observe a single oscillation with these parameters is then about 140. In experimental systems to date, only a few atoms have been trapped near photonic crystals; however, optical depths per atom of $D_{a} \sim 2$ have already been demonstrated [26,27], indicating that sufficient optical depth would be available with about 70 atoms.

While we have focused on the loss of the photonic molecule above, signatures of the molecule and its oscillations can be observed in photon correlation measurements with greatly reduced requirements on the system parameters. In particular, for a weak coherent state input, the normalized second-order correlation function $g^{(2)}(\tau)$ will contain the salient features of the molecular dynamics even with significant two-photon losses, provided that the single-photon component decays in an even stronger manner. This strong single-photon loss can occur by exploiting the design of the correlated transparency window and, in particular, enforcing that the transparency frequency for the two-photon molecule coincides with a region of the single-photon spectrum exhibiting strong absorption. The resulting large loss of the single-photon component leads to the large values of $g^{(2)}(\tau)$ shown in Fig. 5(c), where our simulations include all of the loss mechanisms described above.

\section{SUMMARY AND CONCLUSIONS}

In summary, we have demonstrated that the versatile platform of cold atoms coupled to photonic crystal waveguides leads to new possibilities in quantum optics. In particular, the ability to tune interactions between atoms over distances much greater than the wavelength of light allows the propagation of light through the atomic medium to be highly nonlinear and nonlocal. In contrast with 
current experiments demonstrating nonlinearities due to Rydberg interactions between atoms [9-15], the interactions discussed here can be arranged to give level shifts of the order of the atomic linewidth over the entire atomic ensemble. This allows for correlated transparency of photons through the medium, where depending on the number of photons propagating in the system, the system is only transparent to particular frequencies and spatial configurations. As a result, we have a useful tool to target or engineer certain output states, where the desired state is transparent while others are dissipated out, as we have shown in detail for the case of creating a two-photon molecule. While the focus of our numerical studies here has mainly been in comparing single- and two-photon dynamics in the system, the intuition formed provides important insight into the solution of the multiphoton problem, which is expected to display rich many-body behavior $[18,33]$.

\section{ACKNOWLEDGMENTS}

The authors thank E. Shahmoon, A. Gorshkov, and the Kimble group for stimulating discussions. This work was supported by Fundacio Privada Cellex Barcelona, the MINECO Ramon y Cajal Program, MINECO Severo Ochoa Grant No. SEV-2015-0522, MINECO Plan Nacional Grant CANS, Marie Curie Career Integration Grant ATOMNANO, and ERC Starting Grant FoQAL.

\section{APPENDIX A: OPTICAL DEPTH}

In the main text, we use the form $D=2 N_{a} \Gamma_{1 \mathrm{D}} / \Gamma^{\prime}$ to denote the resonant optical depth for $N_{a}$ two-level atoms. This expression can be derived using the transfer matrix formalism, where linear propagation in one dimension is well described by treating each atom as a refractive element with transmission and reflection coefficients $t=1+r$ and $r=-\Gamma_{1 \mathrm{D}} /\left(\Gamma_{1 \mathrm{D}}+\Gamma^{\prime}\right)$ on resonance $[41,45]$. For $\Gamma_{1 \mathrm{D}} \ll \Gamma^{\prime}$, one expects that reflection is not important, and the transmitted intensity is given by the product of the individual atomic transmittance as $\sim|t|^{2 N_{a}} \sim \exp (-D)$. However, when $\Gamma_{1 \mathrm{D}} \sim \Gamma^{\prime}$, a single atom can act as a strong reflector of light, and the spacing of the atoms becomes crucial in how much light is transmitted through the sample $[41,46]$. In our simulations, we choose the spacing between the atoms $z_{a}$ so that $k_{p} z_{a}=3 \pi / 2$, in which case there is destructive interference between the reflections from each atom in the ensemble. The transmission is then reproduced by $\exp (-D)$ provided $\Gamma_{1 \mathrm{D}} \lesssim 0.2 \Gamma^{\prime}$. Under this condition, the optical properties are the same for two samples with the same $D=2 N_{a} \Gamma_{1 \mathrm{D}} / \Gamma^{\prime}$, and using this property, we can then reduce the number of atoms required to simulate a system with a particular optical depth by increasing $\Gamma_{1 \mathrm{D}}$ proportionally.

The linear properties in the EIT system can be even more robust, remaining the same for constant optical depth even when $\Gamma_{1 \mathrm{D}} \sim \Gamma^{\prime}$. A single three-level atom has transmission and reflection coefficients $t=1+r$ and $r=-\Gamma_{1 \mathrm{D}} \delta /$ $\left[\left(\Gamma_{1 \mathrm{D}}+\Gamma^{\prime}-2 i \delta\right) \delta+2 i \Omega^{2}\right]$ for $\Delta=0$. We then find that after propagating through $N_{a}$ atoms with spacing $k_{p} z_{a}=3 \pi / 2$, the probe field has transmission coefficient given by $\exp \left\{-N_{a} \Gamma_{1 \mathrm{D}} \delta /\left[\left(\Gamma^{\prime}-2 i \delta\right) \delta+2 i \Omega^{2}\right]\right\}$, provided the two-photon detuning $\delta$ satisfies the condition $|\delta| \ll 2|\Omega|^{2} / \Gamma_{1 \mathrm{D}}$. As in the two-level case above, this factor does not depend on $\Gamma_{1 \mathrm{D}}$ and $N_{a}$ individually but on their product. Thus, for probes with $|\delta| \ll 2|\Omega|^{2} / \Gamma_{1 \mathrm{D}}$, we can again reduce the number of atoms and increase $\Gamma_{1 D}$ in a simulation to reduce computational requirements. From a practical point of view, we can check this equivalence in our simulations by changing $\Gamma_{1 \mathrm{D}}$ and $N_{a}$ appropriately and observing that the simulated behavior does not change.

\section{APPENDIX B: EFFECTIVE PROPAGATION OF POLARITONS}

When photons enter the EIT medium, they are converted into polaritons, where the photonic excitation becomes a mixture of a photonic part and spin-wave part with atomic population in the level $|s\rangle$. A single photon entering the system sees the EIT resonance when $\delta=0$ [see Fig. 1(b)]. For more than one photon, the presence of the interaction between the $|s\rangle$ levels shifts the EIT resonance. A shift in the EIT resonance will lead to changes in velocity and dispersion of each pulse, and with a spatially dependent potential as shown in Fig. 4(a), these effects will depend on the separation of polaritons in the media. However, it is not immediately clear how and if the atom-atom interaction potential transforms into an interaction potential for polaritons. To understand more about the system, we now derive an effective propagation equation for polaritons in the system.

To find the effective equation, we model the effective three-level medium in the continuum limit, with linear atomic density $n$, coupled to the probe with slowly varying envelope $\mathcal{E}(t, z)$. In the frame rotating with the input frequency, the system is described by the Hamiltonian [47]

$$
\begin{aligned}
\mathcal{H}= & -n \hbar \int d z\left\{\left(\Delta+i \frac{\Gamma^{\prime}}{2}\right) \sigma_{e e}(z)+\delta \sigma_{s s}(z)\right. \\
& \left.+\left[\sigma_{e s}(z) \Omega e^{i k_{c} z}+\sqrt{\frac{c \Gamma_{1 \mathrm{D}}}{2}} \sigma_{e g}(z) \mathcal{E}(z) e^{i k_{p} z}+\text { H.c. }\right]\right\} \\
& -n^{2} \hbar \int d z d z^{\prime} \sigma_{s s}(z) V\left(z, z^{\prime}\right) \sigma_{s s}\left(z^{\prime}\right),
\end{aligned}
$$

which is identical to the normal Hamiltonian describing EIT systems except for the final term resulting from the atom-atom interactions. The dynamics of the atomic coherences and the probe field are now contained in the respective Heisenberg equations of motion. For a weak probe field, these are 


$$
\begin{aligned}
& \frac{\partial \tilde{\sigma}_{g e}(z)}{\partial t}=i\left(\Delta+i \frac{\Gamma^{\prime}}{2}\right) \tilde{\sigma}_{g e}(z)+i \sqrt{\frac{c \Gamma_{1 \mathrm{D}}}{2}} \mathcal{E}(z)+i \Omega \tilde{\sigma}_{g s}(z), \\
& \frac{\partial \tilde{\sigma}_{g s}(z)}{\partial t}=i \nu(z) \tilde{\sigma}_{g s}(z)+i \Omega^{*} \tilde{\sigma}_{g e}(z), \\
& \frac{\partial \mathcal{E}(z)}{\partial t}+c \frac{\partial \mathcal{E}(z)}{\partial z}=i \sqrt{\frac{c \Gamma_{1 \mathrm{D}}}{2}} n \tilde{\sigma}_{g e}(z),
\end{aligned}
$$

where we have kept only the lowest-order terms in the input field. Here, $\nu(z)=\delta+2 n \int d z^{\prime} \sigma_{s s}\left(z^{\prime}\right) V\left(z, z^{\prime}\right)$ is the detuning of the two-photon transition from $|g\rangle$ to $|s\rangle$, including the modification of the transition frequency due to interactions, while $\tilde{\sigma}_{g e}(z)=\sigma_{g e}(z) e^{-i k_{p} z}$ and $\tilde{\sigma}_{g s}(z)=$ $\sigma_{g s}(z) e^{i\left(k_{c}-k_{p}\right) z}$ are the slowly varying coherences with the rapid phase variation due to the probe and control field propagation removed.

We now transform our equations into the basis of the so-called dark and bright-state polariton operators, $\Psi=\cos \theta \mathcal{E}-\sqrt{n} \sin \theta \tilde{\sigma}_{g s}$ and $\Phi=\sin \theta \mathcal{E}+\sqrt{n} \cos \theta \tilde{\sigma}_{g s}$ $[18,48]$. These are mixtures of the probe field with the atomic coherence, where the mixing angle is given by $\tan \theta=\sqrt{c n \Gamma_{1 \mathrm{D}} /\left(2|\Omega|^{2}\right)}$. The dark-state polariton then propagates with reduced group velocity $v_{g}=c \cos ^{2} \theta$ according to the following equation:

$\frac{\partial \Psi}{\partial t}+v_{g} \frac{\partial \Psi}{\partial z}=i \sin ^{2} \theta \nu(z) \Psi-\cos \theta \sin \theta\left[c \frac{\partial}{\partial z}+i \nu(z)\right] \Phi$.

Their dynamics are coupled to those of the bright-state polariton, given by

$\Phi=\frac{v_{g}}{c|\Omega|^{2}}\left(i \frac{\partial}{\partial t}+\nu(z)\right)\left(i \frac{\partial}{\partial t}+\Delta+i \frac{\Gamma^{\prime}}{2}\right)(\Phi-\tan \theta \Psi)$.

Equations (B3) and (B4) describe both traditional (linear) EIT [48], in the limit where $\nu(z)=\delta$, and the physics of EIT with interactions, where $\nu(z)$ is an operator dependent on the population of state $|s\rangle$. In particular, in the noninteracting case when $\nu(z)=\delta$ lies within the transparency window, the dark-state polariton $\Psi$ decouples from the bright one to lowest order, yielding a propagation without loss for $\Psi$ at the reduced group velocity $v_{g}$. Intuitively, when $\nu(z)$ is an operator, one expects that a transparency condition emerges whenever its expectation value lies within the transparency window. Significantly, it is not necessary for the individual components $\delta, 2 n \int d z^{\prime} \sigma_{s s}\left(z^{\prime}\right) V\left(z, z^{\prime}\right)$ to be small, and thus the possibility emerges for a large number of transparency windows dependent on the combination of detuning $\delta$ and photon number and shape.

To formalize this intuition, we examine the polariton dynamics in the slow-light limit, where $v_{g} \ll c$ and $\sin ^{2} \theta \sim 1$. In this regime, the dark-state polariton is mostly comprised of atomic coherence $\Psi \sim-\sqrt{n} \sigma_{g s}$, while the bright state is comprised mostly of the probe field, and we may replace the atomic operators in the nonlinear detuning by the dark-state polariton operators to get $\nu(z) \approx \delta+2 \int d z^{\prime} \Psi^{\dagger}\left(z^{\prime}\right) V\left(z-z^{\prime}\right) \Psi\left(z^{\prime}\right)$. The bright-state polariton then has a perturbative effect on the propagation of the dark-state polariton when the relevant time dynamics remain within a certain bandwidth (EIT window) around the EIT resonance. In particular, this requires that the polariton has sufficient length $z_{0}$ so that its bandwidth is narrow, $v_{g} / z_{0} \ll|\Omega|^{2} /\left|\Delta+i \Gamma^{\prime} / 2\right|$. At the same time, we also require that the operator $\nu(z)$ leads to values within this window. Specifically, for a particular polariton state $|\psi\rangle$, we estimate the effect of $\nu(z)$ on a polariton at $z$ by evaluating the expectation value $\left\langle\psi\left|\Psi^{\dagger}(z) v(z) \Psi(z)\right| \psi\right\rangle /$ $\left\langle\psi\left|\Psi^{\dagger}(z) \Psi(z)\right| \psi\right\rangle$. This, along with the similar expectation value of the spatial derivative $z_{0} \partial \nu(z) / \partial z$, must fit within the EIT window. In general, $\nu(z)$ must be small and smoothly varying over the length of the pulse, something which is not typically the case for Rydberg interactions, whose spatial variation is large over the length of a polariton with bandwidth within the EIT window.

When these conditions are satisfied, the $\Phi$ terms in Eq. (B3) can be neglected at lowest order, allowing us to replace the time derivatives of $\Psi$ in Eq. (B4) by spatial ones [48]. At this level of approximation, we then find the bright-state dynamics follow those of the dark state according to

$$
\Phi \approx i \sqrt{\frac{v_{g}^{3}}{c \Omega^{4}}}\left(\Delta-\nu(z)+i \frac{\Gamma^{\prime}}{2}\right) \frac{\partial}{\partial z} \Psi .
$$

Substituting Eq. (B5) into Eq. (B3), we find an effective propagation equation for the dark state

$$
\left(\frac{\partial}{\partial t}+v_{g} \frac{\partial}{\partial z}-i \frac{\hbar}{2 m(z)} \frac{\partial^{2}}{\partial z^{2}}\right) \Psi=i \nu(z) \Psi .
$$

Here, the effective mass $m(z)=-\hbar|\Omega|^{2} /\left[\left(2 \Delta_{M}(z)+\right.\right.$ $\left.i \Gamma^{\prime}\right) v_{g}^{2}$ ] depends on the renormalized detuning $\Delta_{M}(z)=$ $\Delta_{L}-2 \int d z^{\prime} \Psi^{\dagger}\left(z^{\prime}\right) V\left(z-z^{\prime}\right) \Psi\left(z^{\prime}\right)$, which in turn depends spatially on the position of other polaritons in the system, where we have identified the control laser detuning $\Delta_{L}=\omega_{L}+\omega_{g s}-\omega_{g e}=\Delta-\delta$.

When the $z$ dependence of the mass is small enough to be neglected, Eq. (B6) is the Heisenberg equation of motion for the dark-state polariton operator that results from the Hamiltonian given in Eq. (1), allowing us to identify this as the effective Hamiltonian for the polaritons. This is clearly true for uniform interactions $V\left(z-z^{\prime}\right)=-U$ in the $N_{p}$-polariton manifold, in which case when the operator $2 \int d z^{\prime} \Psi^{\dagger}\left(z^{\prime}\right) V\left(z-z^{\prime}\right) \Psi\left(z^{\prime}\right)$ multiplies a function of $\Psi\left(z^{\prime}\right)$, 
it can be reduced to the number $-2 U\left(N_{p}-1\right)$. The renormalized detuning becomes $\Delta_{M}=\Delta_{L}+2 U\left(N_{p}-1\right)$, and the effective mass is hence dependent on the number of polaritons in the system. Identifying $\delta=U\left(N_{p}-1\right)$ as the detuning required for $N_{p}$ photon transparency, we can then rewrite this as $\Delta_{M}=\Delta+\delta$.

More generally, the $z$ dependence of the mass can be neglected when the variation of the atomic interaction potential $V\left(z-z^{\prime}\right)$ is small over the region where there is significant polariton population, and we can replace $V\left(z-z^{\prime}\right)$ by a constant. For example, in the photon molecule case, we consider a potential that is approximately quadratic around the natural polariton spacing $r_{0}$, i.e., $V\left(z_{1}-z_{2}\right) \sim V\left(r_{0}\right)+\omega_{M}\left(\left|z_{1}-z_{2}\right|-r_{0}\right)^{2} / z_{0}^{2}$, and consider a molecularlike state where one polariton is centered at $z=0$ and the other near $z=r_{0}$, with Gaussian wave packets $\exp \left(-4 z^{2} / z_{0}^{2}\right)$ and $\exp \left(-4\left(z-r_{0}\right)^{2} / z_{0}^{2}\right)$. In this case, the operator $\Delta_{M}(z)$ acting at the position of the first polariton $(z \sim 0)$ can be approximated by a number $\Delta_{M}(z) \sim \Delta_{L}-2 V\left(r_{0}\right)-\omega_{M}\left(1 / 8+2 z^{2} / z_{0}^{2}\right)$ obtained by integrating the interaction over the wave packet of the second polariton, and similarly at the position of the second polariton. For $\omega_{M} \ll 4\left(\Delta_{L}-2 V\left(r_{0}\right)\right)$, we may take $\Delta_{M} \sim$ $\Delta_{L}-2 V\left(r_{0}\right)$ in the effective mass, which again can be rewritten $\Delta_{M}=\Delta+\delta$ at the molecular transparency $\delta=-V\left(r_{0}\right)$. In the simulations presented in Sec. IV, this approximation is appropriate, where we have that $\omega_{M} \sim 0.1 \Gamma^{\prime}$.

\section{APPENDIX C: INTERATOMIC POTENTIAL}

As described in the main text, the interaction of atoms via a single band edge leads to the effective interaction Hamiltonian $H_{s s}=\left[\hbar\left|\Omega_{s}\right|^{2} g_{c}^{2} /\left(\Delta_{s}^{2} \Delta_{c}\right)\right] \sum_{j, l} \sigma_{s s}^{j} \sigma_{s s}^{l} \times$ $\exp \left(-\left|z_{j}-z_{l}\right| / L\right) E_{k_{b}}^{*}\left(z_{j}\right) E_{k_{b}}\left(z_{l}\right)$. For structures like the "alligator" photonic crystal waveguide described in Ref. [24], the Bloch functions near the band edge are well approximated by the form $E_{k_{b}}(z) \sim \cos \left(k_{b} z\right)$, in which case, if the atoms are trapped at sites commensurate with the band-edge wavelength, we have $E_{k_{b}}(z) \sim 1$.

If the atoms now interact via two distinct band edges, as in Fig. 1(b), the interaction potential $V\left(z_{j}-z_{l}\right)$ becomes the sum of the contributions from the upper $(u)$ and lower $(\ell)$ band edges, $V\left(z_{j}-z_{l}\right)=$ $-\left[\left|\Omega_{s}\right|^{2} / \Delta_{s}^{2}\right]\left[\left(g_{u}^{2} / \Delta_{u}\right) \exp \left(-\left|z_{j}-z_{l}\right| / L_{u}\right)+\left(g_{\ell}^{2} / \Delta_{\ell}\right) \times\right.$ $\left.\exp \left(-\left|z_{j}-z_{l}\right| / L_{\ell}\right)\right]$. The two contributions are each related to effective cavity parameters $g_{u, \ell}, \Delta_{u, \ell}$, and $L_{u, \ell}=\sqrt{2 \alpha_{u, \ell} \omega_{u, \ell} /\left(k_{b}^{2} \Delta_{u, \ell}\right)}$. By adjusting the detunings $\Delta_{u, \ell}$ and band curvatures $\alpha_{u, \ell}$, we can tune the potential to have a minimum at some finite particle separation $r_{0}$, as in Fig. 4(a).

We can now consider the loss introduced into our system for a single atom in the $|s\rangle$ state as a by-product of creating the interatomic potential above. As discussed in the main text (and in more detail in Ref. [31]), the loss due to each band edge is the same as the loss that would result if the interaction were created by a cavity with the same parameters. In particular, in the detuned regime $\left|\Delta_{u, \ell}\right|>\left|g_{u, \ell}\right|$, the loss from spontaneous emission $\Gamma^{\prime}$ and cavity decay $\kappa$ occurs at a rate of approximately $\left[\left|\Omega_{s}\right|^{2} / \Delta_{s}^{2}\right]\left[\Gamma^{\prime}+\kappa g_{u, \ell}^{2} / \Delta_{u, \ell}^{2}\right]$. This loss can then be minimized with respect to the interaction strength $G_{u, \ell}=\left|\Omega_{s}\right|^{2} g_{u, \ell}^{2} /\left(\Delta_{s}^{2} \Delta_{u, \ell}\right)$ by adjusting the detuning, in which case the loss rate from each cavity for an atom in the $|s\rangle$ state becomes $2 G_{u, \ell} / \sqrt{C_{u, \ell}}$ for cavity cooperativity $C_{u, \ell}=g_{u, \ell}^{2} /\left(\kappa \Gamma^{\prime}\right)$.

We can further compare the rate of loss due to these effective cavities to the energy of the two-photon bound state, which gives the rate of oscillation of the photon molecule. The relation between the strengths of the individual potentials due to each band edge and the molecule energy $\omega_{M}$ depends on how the potentials add together and will depend on the desired shape and experimental constraints. As an example, we consider the case where the band edges are arranged such that $G_{u}=G_{\ell} \equiv G$, in which case the contribution from the potential to the dressed state energy of the individual atoms is zero. The potential is now $V\left(z_{j}-z_{l}\right)=$ $G\left[\exp \left(-\left|z_{j}-z_{l}\right| / L_{u}\right)-\exp \left(-\left|z_{j}-z_{l}\right| / L_{\ell}\right)\right]$, and choosing, for example, $L_{\ell}=8 L_{u}$, a potential minimum occurs at $r_{0} \sim 0.3 L_{\ell}$ with depth $\sim 2 G / 3$, which provides an upper limit on the energy of the two-photon molecule energy $\omega_{M}$. Optimizing the detunings as above, we then find that the loss is about $\beta \omega_{M} \sqrt{r_{0} /\left(\lambda C_{\lambda}\right)}$ with $\beta=10$.

The potential we show in Fig. 4(a) has $L_{u}=15 z_{a}$ and $L_{\ell}=30 z_{a}$, where using a smaller ratio between $L_{\ell}$ and $L_{u}$ puts less demands on the engineering of band curvatures since for $G_{u}=G_{\ell}$ we have that $L_{\ell} / L_{u}=\alpha_{\ell} / \alpha_{u}$. The strength of the potential is $G=\left|\Omega_{s}\right|^{2} g_{u, \ell}^{2} /\left(\Delta_{s}^{2} \Delta_{u, \ell}\right) \sim 1.28 \Gamma^{\prime}$, which could be achieved for $\left|\Omega_{s}\right|^{2} / \Delta_{s}^{2} \sim 0.1, g_{u}=\sqrt{2} g_{\ell} \sim 2 \pi \times 3.4 \mathrm{GHz}$, $\Delta_{u}=2 \Delta_{\ell} \sim 2 \pi \times 180 \mathrm{GHz}$, and $\Gamma^{\prime} \sim 2 \pi \times 5 \mathrm{MHz}$. Furthermore the values of $g_{u, \ell}$ and $L_{u, \ell}$ are then consistent with a photonic crystal having $g_{\lambda} \sim 2 \pi \times 12 \mathrm{GHz}$ (such as for the "alligator" structure described in Ref. [31]) and band curvature $\alpha \sim 1$ (similar to that of the structures in Refs. [49] and [50]) for $z_{a} \sim \lambda$.

[1] D. E. Chang, V. Vuletic, and M. D. Lukin, Quantum Nonlinear Optics-Photon by Photon, Nat. Photonics 8, 685 (2014).

[2] Q. A. Turchette, C. J. Hood, W. Lange, H. Mabuchi, and H. J. Kimble, Measurement of Conditional Phase Shifts for Quantum Logic, Phys. Rev. Lett. 75, 4710 (1995).

[3] A. Imamoğlu, H. Schmidt, G. Woods, and M. Deutsch, Strongly Interacting Photons in a Nonlinear Cavity, Phys. Rev. Lett. 79, 1467 (1997). 
[4] L.-M. Duan and H. J. Kimble, Scalable Photonic Quantum Computation through Cavity-Assisted Interactions, Phys. Rev. Lett. 92, 127902 (2004).

[5] K. M. Birnbaum, A. Boca, R. Miller, A. D. Boozer, T. E. Northup, and H. J. Kimble, Photon Blockade in an Optical Cavity with One Trapped Atom, Nature (London) 436, 87 (2005).

[6] S. Haroche and J. M. Raimond, Exploring the Quantum (Oxford University Press, New York, 2006).

[7] H. J. Kimble, The Quantum Internet, Nature (London) 453, 1023 (2008).

[8] A. Reiserer and G. Rempe, Cavity-Based Quantum Networks with Single Atoms and Optical Photons, Rev. Mod. Phys. 87, 1379 (2015).

[9] J. D. Pritchard, D. Maxwell, A. Gauguet, K. J. Weatherill, M. P. A. Jones, and C. S. Adams, Cooperative Atom-Light Interaction in a Blockaded Rydberg Ensemble, Phys. Rev. Lett. 105, 193603 (2010).

[10] Y. O. Dudin and A. Kuzmich, Strongly Interacting Rydberg Excitations of a Cold Atomic Gas, Science 336, 887 (2012).

[11] J. D. Pritchard, K. J. Weatherill, and C. S. Adams, Nonlinear Optics Using Cold Rydberg Atoms, in Annual Review of Cold Atoms and Molecules, edited by K. Madison, Y. Wang, and A. M. Rey (World Scientific Publishing, Singapore, 2013), Vol. 1, pp. 301-350.

[12] T. Peyronel, O. Firstenberg, Q.-Y. Liang, S. Hofferberth, A. V. Gorshkov, T. Pohl, M. D. Lukin, and V. Vuletic, Quantum Nonlinear Optics with Single Photons Enabled by Strongly Interacting Atoms, Nature (London) 488, 57 (2012).

[13] S. Baur, D. Tiarks, G. Rempe, and S. Dürr, Single-Photon Switch Based on Rydberg Blockade, Phys. Rev. Lett. 112, 073901 (2014).

[14] H. Gorniaczyk, C. Tresp, J. Schmidt, H. Fedder, and S. Hofferberth, Single-Photon Transistor Mediated by Interstate Rydberg Interactions, Phys. Rev. Lett. 113, 053601 (2014).

[15] D. Tiarks, S. Baur, K. Schneider, S. Dürr, and G. Rempe, Single-Photon Transistor Using a Förster Resonance, Phys. Rev. Lett. 113, 053602 (2014).

[16] A. V. Gorshkov, J. Otterbach, M. Fleischhauer, T. Pohl, and M. D. Lukin, Photon-Photon Interactions via Rydberg Blockade, Phys. Rev. Lett. 107, 133602 (2011).

[17] O. Firstenberg, T. Peyronel, Q.-Y. Liang, A. V. Gorshkov, M. D. Lukin, and V. Vuletic, Attractive Photons in a Quantum Nonlinear Medium, Nature (London) 502, 71 (2013).

[18] J. Otterbach, M. Moos, D. Muth, and M. Fleischhauer, Wigner Crystallization of Single Photons in Cold Rydberg Ensembles, Phys. Rev. Lett. 111, 113001 (2013).

[19] P. Bienias, S. Choi, O. Firstenberg, M. F. Maghrebi, M. Gullans, M. D. Lukin, A. V. Gorshkov, and H. P. Büchler, Scattering Resonances and Bound States for Strongly Interacting Rydberg Polaritons, Phys. Rev. A 90, 053804 (2014).

[20] M. F. Maghrebi, M. J. Gullans, P. Bienias, S. Choi, I. Martin, O. Firstenberg, M. D. Lukin, H. P. Büchler, and A. V. Gorshkov, Coulomb Bound States of Strongly Interacting Photons, Phys. Rev. Lett. 115, 123601 (2015).

[21] M. Saffman, T. G. Walker, and K. Mølmer, Quantum Information with Rydberg Atoms, Rev. Mod. Phys. 82, 2313 (2010).
[22] J. D. Thompson, T. G. Tiecke, N. P. de Leon, J. Feist, A. V. Akimov, M. Gullans, A. S. Zibrov, V. Vuletić, and M. D. Lukin, Coupling a Single Trapped Atom to a Nanoscale Optical Cavity, Science 340, 1202 (2013).

[23] A. Goban, C.-L. Hung, S.-P. Yu, J. Hood, J. Muniz, J. Lee, M. Martin, A. McClung, K. Choi, D. Chang, O. Painter, and H. Kimble, Atom-Light Interactions in Photonic Crystals, Nat. Commun. 5, 3808 (2014).

[24] S.-P. Yu, J. D. Hood, J. A. Muniz, M. J. Martin, R. Norte, C.-L. Hung, S. M. Meenehan, J. D. Cohen, O. Painter, and H. J. Kimble, Nanowire Photonic Crystal Waveguides for Single-Atom Trapping and Strong Light-Matter Interactions, Appl. Phys. Lett. 104, 111103 (2014).

[25] T. G. Tiecke, J. D. Thompson, N. P. de Leon, L. R. Liu, V. Vuletic, and M. D. Lukin, Nanophotonic Quantum Phase Switch with a Single Atom, Nature (London) 508, 241 (2014).

[26] A. Goban, C.-L. Hung, J. D. Hood, S.-P. Yu, J. A. Muniz, O. Painter, and H.J. Kimble, Superradiance for Atoms Trapped along a Photonic Crystal Waveguide, Phys. Rev. Lett. 115, 063601 (2015).

[27] J. D. Hood, A. Goban, A. Asenjo-Garcia, M. Lu, S.-P. Yu, D. E. Chang, and H. J. Kimble, Atom-Atom Interactions around the Band Edge of a Photonic Crystal Waveguide, arXiv:1603.02771.

[28] G. Kurizki, Two-Atom Resonant Radiative Coupling in Photonic Band Structures, Phys. Rev. A 42, 2915 (1990).

[29] S. John and J. Wang, Quantum Optics of Localized Light in a Photonic Band Gap, Phys. Rev. B 43, 12772 (1991).

[30] E. Shahmoon and G. Kurizki, Nonradiative Interaction and Entanglement between Distant Atoms, Phys. Rev. A 87, 033831 (2013).

[31] J. S. Douglas, H. Habibian, C.-L. Hung, A. V. Gorshkov, H. J. Kimble, and D. E. Chang, Quantum Many-Body Models with Cold Atoms Coupled to Photonic Crystals, Nat. Photonics 9, 326 (2015).

[32] A. González-Tudela, C.-L. Hung, D. E. Chang, J. I. Cirac, and H.J. Kimble, Subwavelength Vacuum Lattices and Atom-Atom Interactions in Two-Dimensional Photonic Crystals, Nat. Photonics 9, 320 (2015).

[33] E. Shahmoon, P. Grisins, H. P. Stimming, I. Mazets, and G. Kurizki, Highly Nonlocal Optical Nonlinearities in Atoms Trapped Near a Waveguide, Optica 3, 725 (2016).

[34] M. Fleischhauer, A. Imamoglu, and J. P. Marangos, Electromagnetically Induced Transparency: Optics in Coherent Media, Rev. Mod. Phys. 77, 633 (2005).

[35] J. D. Joannopoulos, S. G. Johnson, J. N. Winn, and R. D. Meade, Photonic Crystals: Molding the Flow of Light, 2nd ed. (Princeton University Press, Princeton, 2008).

[36] S. John and J. Wang, Quantum Electrodynamics Near a Photonic Band Gap: Photon Bound States and Dressed Atoms, Phys. Rev. Lett. 64, 2418 (1990).

[37] G. Agarwal, S. Gupta, and R. Puri, Fundamentals of Cavity Quantum Electrodynamics (World Scientific, Singapore, 1995).

[38] S. Bay, P. Lambropoulos, and K. Mølmer, Atom-Atom Interaction in Strongly Modified Reservoirs, Phys. Rev. A 55, 1485 (1997). 
[39] P. Lambropoulos, G. M. Nikolopoulos, T. R. Nielsen, and S. Bay, Fundamental Quantum Optics in Structured Reservoirs, Rep. Prog. Phys. 63, 455 (2000).

[40] E. Shahmoon, I. Mazets, and G. Kurizki, Non-additivity in Laser-Illuminated Many-Atom Systems, Opt. Lett. 39, 3674 (2014).

[41] D. E. Chang, L. Jiang, A. V. Gorshkov, and H. J. Kimble, Cavity QED with Atomic Mirrors, New J. Phys. 14, 063003 (2012).

[42] M. Fleischhauer and M. D. Lukin, Dark-State Polaritons in Electromagnetically Induced Transparency, Phys. Rev. Lett. 84, 5094 (2000).

[43] T. Caneva, M. T. Manzoni, T. Shi, J. S. Douglas, J. I. Cirac, and D. E. Chang, Quantum Dynamics of Propagating Photons with Strong Interactions: A Generalized InputOutput Formalism, New J. Phys. 17, 113001 (2015).

[44] B. R. Mollow, Pure-State Analysis of Resonant Light Scattering: Radiative Damping, Saturation, and Multiphoton Effects, Phys. Rev. A 12, 1919 (1975).

[45] D. E. Chang, A. H. Safavi-Naeini, M. Hafezi, and O. Painter, Slowing and Stopping Light Using an
Optomechanical Crystal Array, New J. Phys. 13, 023003 (2011).

[46] D. E. Chang, A. S. Sorensen, E. A. Demler, and M. D. Lukin, A Single-Photon Transistor Using Nanoscale Surface Plasmons, Nat. Phys. 3, 807 (2007).

[47] M. Hafezi, D. E. Chang, V. Gritsev, E. Demler, and M. D. Lukin, Quantum Transport of Strongly Interacting Photons in a One-Dimensional Nonlinear Waveguide, Phys. Rev. A 85, 013822 (2012).

[48] M. Fleischhauer and M. D. Lukin, Quantum Memory for Photons: Dark-State Polaritons, Phys. Rev. A 65, 022314 (2002).

[49] C.-L. Hung, S. M. Meenehan, D. E. Chang, O. Painter, and H. J. Kimble, Trapped Atoms in One-Dimensional Photonic Crystals, New J. Phys. 15, 083026 (2013).

[50] X. Zang, J. Yang, R. Faggiani, C. Gill, P. G. Petrov, J.-P. Hugonin, K. Vynck, S. Bernon, P. Bouyer, V. Boyer, and P. Lalanne, Interaction between Atoms and Slow Light: A Study in Waveguide Design, Phys. Rev. Applied 5, 024003 (2016). 